\title{
New-onset diabetes after kidney transplantation revealing HNF1B-associated disease
}

\author{
Ana M Lopes $\mathbb{D}$ and Sofia Teixeira
}

Endocrinology Department, Centro Hospitalar e Universitário do Porto, EPE, Porto, Portugal

Correspondence should be addressed to A M Lopes

Email

analopes09@gmail.com

\section{Summary}

Molecular alterations of the transcription factor hepatocyte nuclear factor 1B (HNF1B) are associated with systemic disease, with kidney disease and maturity-onset diabetes of the young (MODY) as the most characteristic manifestations. Other features comprise pancreatic exocrine insufficiency, liver and biliary anomalies, and genital tract malformations. HNF1B-associated disease is clinically heterogeneous, and therefore the diagnosis is challenging. The authors describe the case of a 19-year-old man with new-onset diabetes after kidney transplantation (NODAT). The kidney disease presented during fetal life as bilateral hyperechogenic kidneys. Renal function progressively deteriorated during childhood, and at the age of 19, the patient was submitted to a living-kidney transplant. Two weeks after transplant, NODAT developed. Given the young age and normal body weight, NODAT was unexpected, and the possibility of HNF1B-associated disease was considered. Screening for mutations in HNF1B was undertaken, and a known mutation was found. As this case highlights, HNF1B-associated disease should be considered when NODAT unexpectedly develops in young kidney transplant recipients with a suggestive renal disease.

\section{Learning points:}

- $\quad H N F 1 B$ anomalies are associated with systemic disease, including kidney disease, diabetes mellitus, pancreatic exocrine insufficiency, liver test abnormalities and genital tract malformations.

- Phenotype is variable and there are no pathognomonic manifestations, but kidney disease appears to be the most common feature and diabetes the most frequent extra-renal phenotype.

- Spontaneous gene alterations are common, and the lack of family history should not exclude the diagnosis.

- HNF1B defects should be considered when NODAT develops in a young adult kidney transplant recipient with a suggestive kidney disease and without extensive risk factors for diabetes.

- The most appropriate treatment for HNF1B-associated diabetes is not established, but immunosuppressive therapy superimposed on a beta-cell dysfunction seems to determine the need for insulin therapy after a variable period.

- Immunosupressive regimens free of calcineurin inhibitors should be considered in patients with HNF1B-associated disease to minimize the risk of developing NODAT.

\section{Background}

Since the first description of an $H N F 1 B$ mutation associated with early-onset diabetes in 1997, the knowledge of $H N F 1 B$ genetic changes has evolved significantly, and it is currently known that HNF1B anomalies are associated with a systemic disease with marked phenotypic variability (1). The diagnosis of HNF1B-associated disease is challenging, 
and many cases likely remain undetected or misdiagnosed. The authors describe the case of a young male with a previously unrecognized HNF1B mutation that developed NODAT after kidney transplantation, which triggered the diagnosis of HNF1B-associated disease.

\section{Case presentation}

A 19-year-old man was referred to our clinic because of new-onset diabetes after kidney transplantation (NODAT). Two weeks after transplantation, routine blood analysis revealed marked hyperglycemia (up to 17.2 $\mathrm{mmol} / \mathrm{L}$ ). He denied catabolic symptoms, and there was no evidence of ketoacidosis. Through childhood, blood glucose levels were normal, with fasting blood glucose levels ranging from 4.2 to $5.2 \mathrm{mmol} / \mathrm{L}$, with no trend of deterioration over time. His body weight was $55 \mathrm{~kg}$ and BMI $21.5 \mathrm{~kg} / \mathrm{m}^{2}$. Kidney disease was suspected on a routine fetal ultrasonographic scan (US) performed at 20 weeks' gestation, revealing bilateral hyperechogenic kidneys. After birth, the US demonstrated hyperechogenic kidneys with small cysts, and plasma creatinine was abnormally elevated. The patient was the first child of non-consanguineous and healthy parents, which pointed to autosomal recessive polycystic kidney disease (ARPKD). Thereafter, renal function progressively deteriorated. By the age of 18, plasma creatinine level was $433.2 \mu \mathrm{mol} / \mathrm{L}$ (corresponding to a glomerular filtrate rate (GFR) of 15.9 $\mathrm{mL} / \mathrm{min} / 1.73 \mathrm{~m}^{2}$ ). One year later, the patient received a living kidney transplant from his 52-year-old father. There was no morphological abnormality on the father's kidney, and his renal function was normal. The procedure was complicated by partial graft necrosis. At hospital discharge, plasma creatinine level was $185.6 \mu \mathrm{mol} / \mathrm{L}$ (GFR: $44.3 \mathrm{~mL} /$ $\left.\min / 1.73 \mathrm{~m}^{2}\right)$. At that time, the immunosuppressive regimen comprised tacrolimus $15 \mathrm{mg} /$ day, mycophenolate mofetil $2000 \mathrm{mg} /$ day, and prednisolone $15 \mathrm{mg}$ /day.

\section{Investigation}

Given the patient's young age and normal body weight, and the absence of other major risk factors, the development of NODAT was unexpected. A history of diabetes was noted in the patient's maternal grandmother and uncle, with no other known relevant family history, including kidney disease (Fig. 1). Fasting C-peptide was $1.3 \mathrm{nmol} / \mathrm{L}$ (normal range: $0.4-1.5 \mathrm{nmol} / \mathrm{L}$ ). Islet autoantibodies, including GADA, ICA, IA-2A, and ZnT8A, were all negative. US pancreatic evaluation was normal. The association of diabetes with such kidney morphological

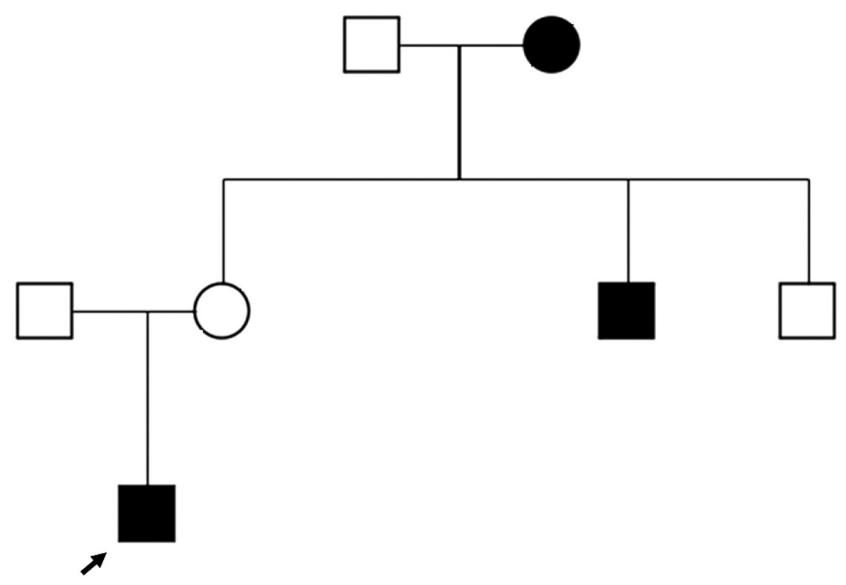

Figure 1

Family pedigree of the patient showing the affected patient (proband) and other affected family members with diabetes.

abnormalities raised the possibility of maturity-onset diabetes of the young (MODY) type 5 and hepatocyte nuclear factor 1B (HNF1B) gene defects. Screening for mutations in the $H N F 1 B$ gene was undertaken and a known missense heterozygous mutation (p.S148L, c.443 C>T) in exon 2 was found. So far, it was not possible to perform genetic testing on any other family member, but the family was referred for genetic counseling. Regarding other manifestations, the patient had no complaints suggestive of exocrine dysfunction, liver enzymes were normal over the years (both alanine aminotransferase and aspartate aminotransferase persistently below the upper limit of the normal range, both before and after transplantation), and there was no clinical evidence of genital tract malformations.

\section{Treatment}

Insulin therapy was started with a long-acting analog (0.16 $\mathrm{UI} / \mathrm{kg} /$ day). The insulin dose was progressively reduced, following reductions in tacrolimus and prednisolone doses. Five months after NODAT diagnosis, insulin was switched to a dipeptidyl peptidase 4 (DPP-4) inhibitor. At that time, the patient was under tacrolimus $8 \mathrm{mg} /$ day and prednisolone $5 \mathrm{mg} /$ day. However, the patient stopped it a few weeks later due to vomiting. As glycated hemoglobin (HbA1c) remained below 6.5\%, the patient was kept without medication. However, 16 months later, HbA1c increased to $6.9 \%$. Therefore, sitagliptin $25 \mathrm{mg} /$ day was resumed, with good tolerance. Nevertheless, HbA1c progressively increased to $8 \%$ in the following 8 months (Fig. 2). Renal function slowly deteriorated, with plasma creatinine level ranging between 185.6 and 


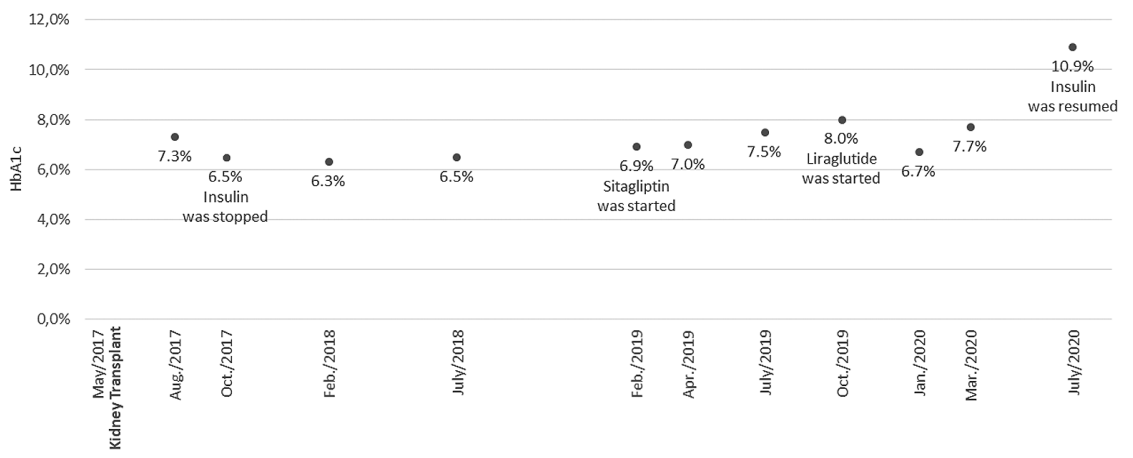

Figure 2

Evolution of the patient's glycemic control after NODAT diagnosis.
$229.8 \mu \mathrm{mol} / \mathrm{L}$ (GFR range: $34-44 \mathrm{~mL} / \mathrm{min} / 1.73 \mathrm{~m}^{2}$ ). At that time, the patient was under tacrolimus $6 \mathrm{mg} /$ day and prednisolone $5 \mathrm{mg} /$ day. After discussing the therapeutic options, therapy was switched to liraglutide, which was progressively titrated to $1.8 \mathrm{mg} /$ day.

\section{Outcome and follow-up}

Two months after liraglutide introduction, HbA1c dropped to $6.7 \%$. However, glycemic control again deteriorated, and HbA1c increased up to 10.9\% (Fig. 1). Immunosuppressive therapy doses were unchanged, but the patient reported less physical activity. Plasma creatinine level was $224.5 \mu \mathrm{mol} / \mathrm{L}$, corresponding to a GFR of $35.2 \mathrm{~mL} / \mathrm{min} / 1.73 \mathrm{~m}^{2}$. At this time, liraglutide was stopped, and insulin with a long-acting analog (0.14 UI/ $\mathrm{kg}$ /day) was resumed. Two months later, glycemic control slightly improved. Insulin therapy will be maintained, and treatment management will continue as considered appropriate.

\section{Discussion}

HNF1B is involved in the embryogenesis and tissuespecific gene expression of several organs, including the kidney, pancreas, liver, and genital tract. Even though HNF1B mutations were first associated with MODY, it is currently known that $H N F 1 B$ anomalies result in a systemic disease that follows the expression of HNF1B (1). The most frequently described manifestations include kidney disease, early-onset diabetes, pancreatic exocrine insufficiency, liver dysfunction, and genital tract malformations. Notably, there is marked phenotypic variability, suggesting that other genetic or environmental factors might modify the pattern and severity of the disease (1).

The prevalence of HNF1B-associated disease is unknown. The mean overall detection rate of HNF1B defects in cohorts of patients with kidney disease was $19 \%$
(1). By contrast, they are a rare cause of MODY, accounting for less than $1 \%$ of the cases (1). As most cohorts were preselected for kidney disease and/or diabetes, the frequency of these and other clinical features is unclear.

Genetic changes in the $H N F 1 B$ gene comprise either heterozygous intragenic mutations or gene deletions. Although HNF1B-associated disease is inherited in an autosomal-dominant manner, spontaneous genetic changes are common, which explains the frequent absence of a family history of kidney disease or diabetes (1). Given the family history of diabetes in this patient, we hypothesize that the mutation was inherited.

Kidney disease appears to be the most common manifestation, but considerable variation is found in its phenotype and severity $(2,3,4)$. Cystic disease is the predominant morphological manifestation in both children and adults $(2,3)$. As observed in this patient, kidney disease can present during fetal life as bilateral hyperechogenic kidneys of normal or slightly increased size (5). Tubular transport abnormalities can also be present, and hypomagnesemia is a common and distinctive feature $(1,3)$. Hyperuricemia and early-onset gout are also observed. Importantly, renal function is frequently impaired, and up to $20 \%$ of patients progress to end-stage renal disease (3).

Given the role of HNF1B in pancreatic development, HNF1B-associated disease can present with pancreatic hypoplasia, and both endocrine and exocrine dysfunction can occur (1). Diabetes is considered as the most frequent extra-renal phenotype. In the largest cohort described to date (3), Dubois-Laforgue et al. reported diabetes in $82 \%$ of 194 patients. Diabetes is usually of early-onset (median age 20 years), but it can be found at any age from the neonatal period to late middle age (1). Clinical presentation seems highly variable, including ketoacidosis (3). The pathophysiology of HNF1B-associated diabetes has not been definitively established. Both beta-cell dysfunction and reduced hepatic insulin sensitivity have been implicated (1). Beta-cell dysfunction, resulting 
in reduced insulin secretion, may be a consequence of pancreatic hypoplasia and beta-cell mass reduction. However, primary functional abnormalities in insulin secretion must also occur, as pancreatic hypoplasia and endocrine dysfunction do not completely correlate (6).

As this case demonstrates, HNF1B-associated diabetes can present as NODAT. In this patient, it was the association of an unexpected NODAT and the kidney disease that triggered the diagnosis. The frequency of NODAT in HNF1B-associated disease is unknown. It is suggested that $H N F 1 B$ defects determine a genetic susceptibility to NODAT, as the reduced insulin secretory capacity of betacell is unmasked by the immunosuppressive drugs $(7,8)$. Faguer et al. (9) hypothesized that immunosuppressive therapy may switch off residual $H N F 1 B$ expression and thus potentiate beta-cell dysfunction. Combining clinical data and in vitro experiments, these authors suggested that calcineurin inhibitors (CNI) induce a downregulation of the nonmutated allele, mimicking $H N F 1 B$ biallelic inactivation. In this patient, given the deterioration of renal function, immunosuppressive regimens avoiding CNI were not considered. However, considering that immunosuppressive regimens free of CNI may minimize the deterioration of NODAT, if the patient's glycemic control remains above the target, the possibility of such a regiment will be discussed. Whether all kidney transplant recipients will ultimately develop diabetes is unknown. Interestingly, Waller et al. (8) reported the case of a boy with a previously unrecognized $H N F 1 B$ mutation in which NODAT regressed after transplant nephrectomy and cyclosporine and steroid withdrawal. It is not clear whether NODAT has a distinctive presentation in patients with HNF1B-associated disease. Some authors highlighted its very early onset after transplantation, as occurred in this case, but this was not seen in all patients reported.

The most appropriate treatment for HNF1B-associated diabetes is not established. In keeping with the hypothesis of beta-cell dysfunction, the majority of patients are treated with insulin (3). However, residual insulin secretion has been demonstrated, and some authors argue that insulin treatment can be delayed for a few years (3). The choice for a DPP-4 inhibitor was based on the patient characteristics (normal weight and post-transplantation kidney status) and an only slightly elevated HbA1c value. Also, there was the aim of simplifying the therapeutic regimen, switching to an oral agent. Even though our patient remained without any treatment for more than a year, glycemic control eventually deteriorated. The initial response to liraglutide was impressive, but glycemic control worsened again, and insulin therapy was required
6 months later. We hypothesize that the patient's young age and normal body weight, in addition to the absence of major pancreatic morphological abnormalities, contributed to the initial glycemic control. However, the immunosuppressive therapy possibly limited an extended response to non-insulin agents, and so insulin therapy was resumed nearly 3 years after diabetes diagnosis. As far as we know, there is no data on treatment of HNF1Bassociated diabetes with glucagon-like peptide-1 receptor agonists. Whether patients with HNF1B-associated diabetes not receiving calcineurin inhibitors respond to these agents remains to be elucidated.

Other reported manifestations of HNF1B-disease include abnormal liver function, usually manifesting as an asymptomatic rise in liver enzymes; biliary anomalies presenting as bile duct cysts; and various genital tract malformations (3). Of note, the clinical spectrum of the disease seems to be expanding $(1,6)$. At this time, there is no evidence of any of these additional features in this patient.

In conclusion, HNF1B-associated disease is remarkable for its marked phenotypic variability and the high rate of de novo cases. The diagnosis is challenging and many cases likely remain undetected or misdiagnosed. However, the right diagnosis is essential to provide the best care to both patients and their relatives. We believe the most important step for diagnosis of HNF1B-associated disease is to first recognize this possibility. Findings such as hypomagnesemia or pancreatic structural anomalies can support the indication for genetic testing (10). Additionally, an HNF1B score using a weight combination of clinical features has been proposed for selecting patients for HNF1B testing (4). Finally, as the case we present highlights, HNF1B-associated disease should be considered when NODAT unexpectedly develops in young kidney transplant recipients with a suggestive kidney disease. Even though the most appropriate treatment for HNF1Bassociated diabetes is not established, immunosuppressive therapy superimposed on a beta-cell dysfunction seems to determine the need for insulin therapy after a variable period.

\section{Declaration of interest}

The authors declare that there is no conflict of interest that could be perceived as prejudicing the impartiality of this case report.

\section{Funding}

This research did not receive any specific grant from any funding agency in the public, commercial or not-for-profit sector. 


\section{Patient consent}

Written informed consent has been obtained from the patient for publication of the submitted article and accompanying images.

\section{Author contribution statement}

Both authors were involved in the management of the patient, and in the writing and editing of the manuscript.

\section{References}

1 Clissold RL, Hamilton AJ, Hattersley AT, Ellard S \& Bingham C. HNF1B-associated renal and extra-renal disease - an expanding clinical spectrum. Nature Reviews: Nephrology 201511 102-112. (https://doi.org/10.1038/nrneph.2014.232)

2 Faguer S, Decramer S, Chassaing N, Bellanné-Chantelot C, Calvas P, Beaufils S, Bessenay L, Lengelé JP, Dahan K, Ronco P, et al. Diagnosis, management and prognosis of $H N F 1 B$ nephropathy in adulthood. Kidney International 201180 768-776. (https://doi.org/10.1038/ ki.2011.225)

3 Dubois-Laforgue D, Cornu E, Saint-Martin C, Coste J, BellannéChantelot C, Timsit J \& Monogenic Diabetes Study Group of the Société Francophone du Diabète. Diabetes, associated clinical spectrum, long-term prognosis and genotype/phenotype correlations in 201 adult patients with hepatocyte nuclear factor 1B (HNF1B) molecular defects. Diabetes Care 201740 1436-1443. (https://doi. org/10.2337/dc16-2462)

4 Faguer S, Chassaing N, Bandin F, Prouheze C, Garnier A, Casemayou A, Huart A, Schanstra JP, Calvas P, Decramer S, et al. The HNF1B score is a simple tool to select patients for HNF1B gene analysis. Kidney International 201486 1007-1015. (https://doi. org/10.1038/ki.2014.202)

5 Heidet L, Decramer S, Pawtowski A, Morinière V, Bandin F, Knebelmann B, Lebre AS, Faguer S, Guigonis V, Antignac C, et al. Spectrum of $H N F 1 B$ mutations in a large cohort of patients who harbour renal diseases. Clinical Journal of the American Society of Nephrology 20105 1079-1090. (https://doi.org/10.2215/CJN.06810909)

6 Kettunen JLT, Parvianinen H, Miettinen PJ, FärKKilä M, Tamminen M, Salonen P, Lantto E \& Tuomi T. Biliary anomalies in patients with HNF1B diabetes. Journal of Clinical Endocrinology and Metabolism 2017 102 2075-2082. (https://doi.org/10.1210/jc.2017-00061)

7 Kanda S, Morisada N, Kaneko N, Yabuuchi T, Nawashiro Y, Tada N, Nishiyama K, Miyai T, Sugawara N, Ishizuka K, et al. New-onset diabetes after renal transplantation in a patient with a novel HNF1B mutation. Pediatric Transplantation 201620 467-471. (https://doi. org/10.1111/petr.12690)

8 Waller SC, Rees L, Woolf AS, Ellard S, Pearson ER, Hattersley AT \& Bingham C. Severe hyperglycemia after renal transplantation in a pediatric patient with a mutation of the hepatocyte nuclear factor- $1 B$ gene. American Journal of Kidney Diseases 200240 1325-1330. (https://doi.org/10.1053/ajkd.2002.36915)

9 Faguer S, Esposito L, Casemayou A, Pirson Y, Decramer S, Cartery C, Hazzan M, Garrigue V, Roussey G, Cointault O, et al. Calcineurin inhibitors downregulate HNF-1B and may affect the outcome of HNF1B patients after renal transplantation. Transplantation 2016100 1970-1978. (https://doi.org/10.1097/TP.0000000000000993)

10 Madariaga L, García-Castaño A, Ariceta G, Martínez-Salazar R, Aguayo A, Castaño L \& Spanish Group for the Study of HNF1B Mutations. Spanish group for the study of HNF1B mutations. Variable phenotype in HNF1B mutations: extrarenal manifesations distinguish affected individuals from the population with congenital anomalies of the kidney and urinary tract. Clinical Kidney Journal 201912 373-379. (https://doi.org/10.1093/ckj/sfy102)

Received in final form 2 December 2020

Accepted 7 January 2021 